Research Article

Nada I. Najim*

\title{
Improving Data Delivery in Large Environments and Mobile Areas
}

https://doi.org/10.1515/comp-2020-0211

Received Feb 17, 2020; accepted Aug 13, 2020

Abstract: Advances in communication technology have significantly altered our knowledge about telephone networks. The remarkable expansion in communication is obvious in numerous aspects of life of life; the transmission of information has never been easier. Wireless communication has also had a remarkable development, and these facilities have become more reliable and easily accessible.

This study, through a descriptive approach in accordance with earlier studies, aims to explain how to increase the capacity of wireless data delivery in addition to other important challenges that face the enhancement of these services in remote areas. This study highlights the challenges that accompany the adoption of wireless networks in remote areas: particularly signal strength. This is highly linked to the specific features of rural areas in terms of living habits in separated nodes rather than in high-density population centers areas.

Keywords: signal strength, data delivery, data delivery in remote area, mobile area

\section{Introduction}

The unescapable introduction and continuous development of advanced technology in almost all fields of life has changed the way most facilities are run; now, centralized high-performance computers are responsible for monitoring and recognizing people in vital establishments such as airports, city centers, and so on. These computers rely on a diverse and complex highly secured network to send and receive data [1-3].

The era of information technology in which we now live is obliged to involving technology in each sector of life: education, medicine, agriculture, commerce, entertainment, and even critical fields such as military and more. The ac-

\footnotetext{
*Corresponding Author: Nada I. Najim: Computer Engineering Department, College of Engineering, University of Mosul, Mosul, 41000, Iraq; Email: nadaalmaaroof@gmail.com
}

๑ Open Access. (C) 2021 N. I. Najim, published by De Gruyter. (cc) BY License celerated advances in mobile-device technology, such as laptops and smart phones, with the emergence of the internet have further broadened their impact on human life [4].

Modern technology, especially mobile phones, aid in the communication between friends or family; people can simply access social network sites online with their smart phones. Thanks to the internet, all types of data-text messages, photos, or videos-can be sent and received between geographically dispersed individuals easily. However, to achieve smooth connection of devices to networks, an appropriate signal strength is required $[5,6]$.

One component of an effective wireless network is the ability to transfer a large amount of data, such as multimedia file, with minimal energy requirement and noise distortion, and an appropriate response time [7, 8].

This study aims to clarify how to build a network capable of sending and receiving data in a relatively large environment where communication is weak.

\section{The study problem}

The over-reliance on mobile devices has been widely observed. The most common means of communication online are as follows: sending and receiving various types of data online (messages, photos, high-resolution videos, and so on). The most frequent problems encountered by this sector (i.e. the problems regarding communication using mobile devices) are network capacity and the quality of connection. These are linked to various factors such as frequency range and distance to the nearest booster towers in the region and many more.

The problem this study aims to solve is how to increase the capacity of networks to transmit and receive data in areas where the connection is weak, and improve the response time of data being sent to mobile phones.

\subsection{The importance of studying}

Mobile devices are considered the most frequent alternatives to ordinary computers nowadays. They displace the use of computers in various settings such as at home and 
educational institutions. Needless to say, they have an influence as an educational tool and in the entertainment field as well. Not only do ordinary people and students use them, but mobile devices are also considered vital for scientists and professionals alike. Their ease of use and their ability to access the internet enables anyone to perform business and interact with others online anywhere in the world: both cheaply and easily. In addition to this, they influence people's social interactions and has been used as a medium to melt the borders across cultures.

However, using mobile devices to access information online in some areas is rather difficult. This is because of the poor signal strength in those areas. The goal of this study is to explore how to build an effective system capable of increasing the signal strength in networks and thus improve the reliability and response time of these networks.

\subsection{Objectives of the study}

The objectives of the study can be defined as follows:

- To identify how to increase signal strength in wireless networks.

- To identify the significant challenges in the establishment and development of telecommunications and information technology in rural and remote areas.

\section{Related work}

\section{Increase signal strength in networks}

Mudumbai [9] explained that a key problem of communication in wireless networks is in enhancing the signal enhancing the signal strength even with spatial diversity to achieve reliability in communication. The research aims to demonstrate how to make use of spatial diversity and the available frequency in networks to achieve efficiency in communications. The sensors were used because of their two main Characteristics: first, increasing the system's signal reception; second, their excellent compatibilities with analysis and simulation, which overall provides important feedback for the sensors [9].

Voigt et al. [10] also noted that many techniques create networks of low energy in wide-ranging areas, though with a lot of problems. In this work, they studied the Long Range (LoRa) Low-Power Wide-Area Network (LPWAN) in terms of their ability to increase signal strength in the receivers without increasing the cost. The results showed that the use of these techniques and protocols can improve the performance of the network with the use of multiple base stations.
This clearly showed that this method outperforms the use of directional antennas [10].

\section{Network Data Delivery Service (NDDS)}

Pardo-Castellote and Schneider [11] mentioned the new NDDS network data exchange system that relies mainly on information producers (sourcing) and consumers (banks). The purpose of this research was to describe the NDDS system in detail. The NDDS has a unique platform capable of exchanging data and information among applications that are distributed on a computer network. It also provides new foundation capabilities for distributed control systems that use NDDS as their standard communication mechanism. This is also applied to several applications that depend on NDDS to communicate between modules such as those used to create an integrated platform in a robotic cell work arm [11]. Furthermore, Almadani [12] noted that the NDDS system is one of the most appropriate systems in terms of design and construction at acceptable costs and maintenance. Needless to say, they offer the build of a reusable system with the least efforts, the best performance, and the least expenses [12].

\section{Challenges and difficulties in the development of telecommunications and information technology in far and remote areas}

Adediran et al. [13] mentioned access to wireless networks in rural areas as a challenge for many rural communities. This is because of the primitive infrastructure in these areas. This study aimed to highlight the most important challenges that contribute to this situation such as poverty, the lack of proper infrastructure and social services, low level of education, and road surfacing and electricity production. Other factors worsen the situation and necessitate higher financial support such as the distance to the nearest civil centers and the separation of end-users over wide areas. The study urged the need to provide solutions to the expanded coverage nationwide, provide robust infrastructure, and encourage the manufacture of local telecommunication devices [13].

Dubale [14] also pointed out these challenges and included others such as the lack of physical capacity, lack of technical and economic skills, and lack of adequate infrastructure development (roads and electricity infrastructure), in addition to the lack of the host's core technical capacity to handle and operate the terminal [14]. 


\section{Increase signal strength in the networks}

Networks are useful in their ability to provide communication in crowded and isolated places, as well as the ability to provide communications for mobile users. Therefore, it is important to study the elements that have a detrimental effect on signal strength in wireless networks. Examples of these influences can be found in $[14,15]$.

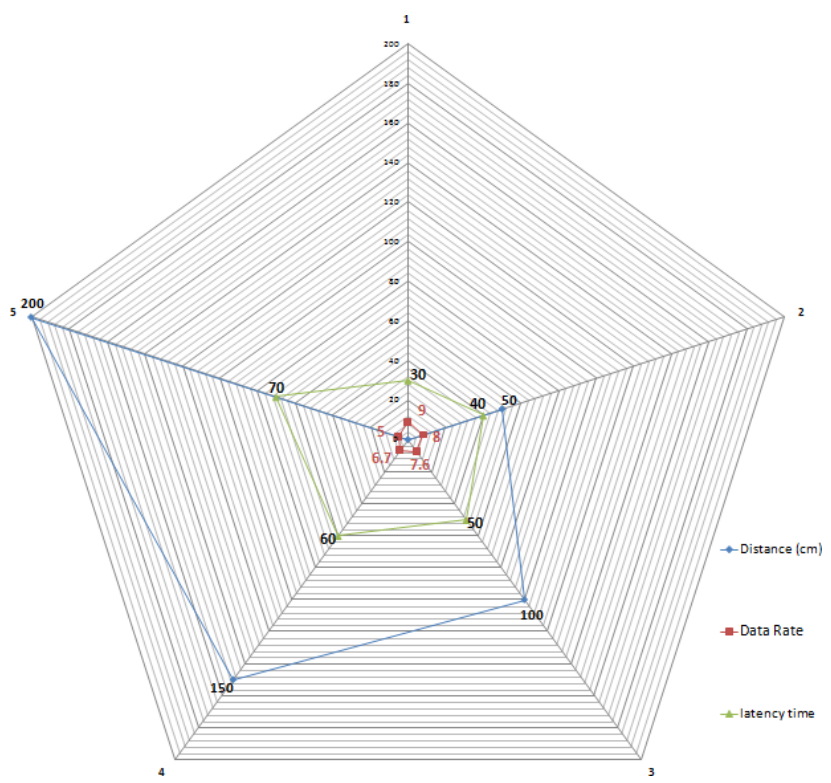

(a) Effect of distance over data rate and the latency time

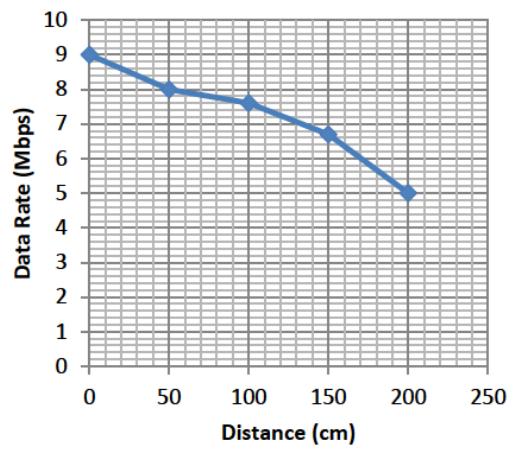

(b) Relationship between distance and data rate

Figure 1: (a) \& (b) Shows that the greater the distance from the point of access, the lower the data rate and the greater the latency time, as expected.

\subsection{Location}

Location is very important because it plays a key role in communication and signal strength. The greater the distance from the Access Point, the lower the signal strength (as shown in Figures $1 \& 2$ ). In addition to this, the greater the natural barriers between the access point and the user, the weaker signal strength received. Therefore, the importance of location comes from its direct impact on the quality of connection [17, 18].

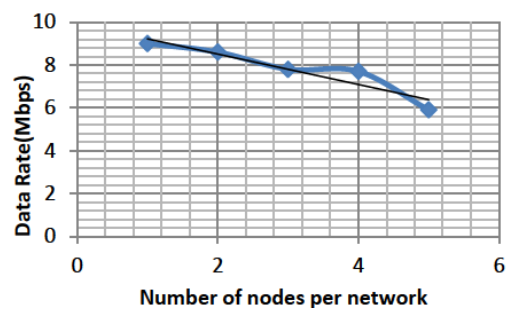

Figure 2: The greater the number of nodes per network, the lower the data rate for the same distance (for distance of $200 \mathrm{~cm}$.

\subsection{Barriers and contraventions}

These are the physical metal and stone barriers that affect the router connectivity significantly and may weaken and/or cut off the connection permanently. Therefore, there should not be an access point away from the barriers so as not to cause weakness in the router capabilities (as shown in Figure 3).

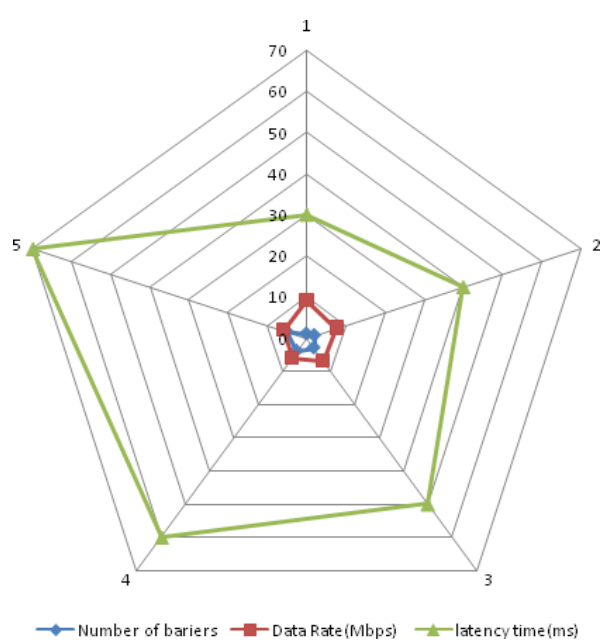

Figure 3: The greater the number of barriers away from an access point, the lower the data rate and the greater the latency time for the same distance. 


\subsection{Change antenna}

Each access point must have an antenna, a one or two directional-Omni, each of which forms a full-circle coverage radius, i.e. the signal can be strong enough to reach the whole house and the adjacent rooms [19].

\subsection{Wireless repeater}

A repeater can be used to extend the network coverage indoors; while experiencing poor signal In an area, the repeater, rather than an additional Access Point, will improve the signal strength and keep the error-related-to-networksinteractions rate to the minimum.

\subsection{Update the $11.802 \mathrm{~b}$ protocol to $11.802 \mathrm{~g}$}

The $11.802 \mathrm{~b}$ was the most prevalent technology worldwide before the $11.802 \mathrm{~g}$ was invented. The latter is five times faster than the 11.802b. Both technologies use the same range of frequency $(2.4 \mathrm{MHz})$ and they are compatible to some extent. However, $11.802 \mathrm{~g}$ requires an upgraded access point to deal with the higher performance of $11.802 \mathrm{~g}[20,21]$.

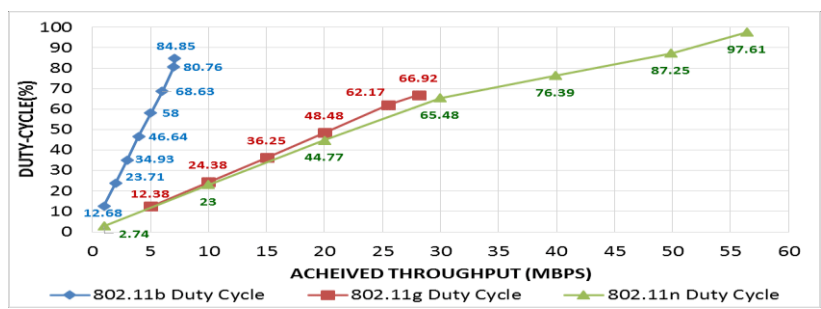

Figure 4: $802.11 \mathrm{~b} / \mathrm{g} / \mathrm{n}$ single pair duty cycle vs. throughput.

\section{Network data delivery service (NDDS)}

The Network Data Delivery Service (NDDS) system builds on the model of information producers and consumers; the producer registers a set of data instances unaware of prospective consumers. In the NDDS, a subscription-based model is used to reduce the overhead required by clientserver architecture. See Figure 5.

The NDDS identifies data instances by name; the scope of this name extends to all the tasks sharing data. If two

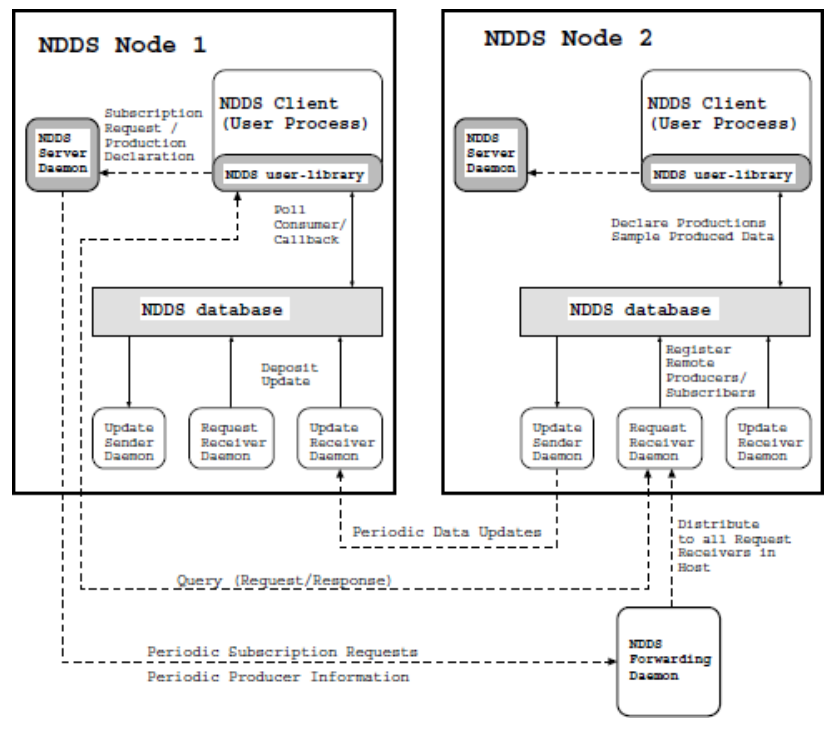

Figure 5: Communication between NDDS nodes

data instances must be distinguished by any NDDS client, they must be given a different NDDS name and requires all data instances to be of a known type [11, 12]

\section{Challenges of telecommunications and information technology in remote areas}

Remote areas are characterized by their relatively small population compared to urban areas. These areas are usually dependent on agricultural and comparatively lesser technical activities. This has created some difficulties in promoting wireless networks in these areas [22]:

1. Access problems due to distance, transport networks, and isolation;

2. Lack of robust infrastructure;

3. Lack of adequate telecommunication infrastructure;

4. High cost of physical access and installation of equipment;

5. Low geographical density of the target population (i.e. inhabitants of small villages in geographically separated populations);

6. Low income and higher poverty rate;

7. Higher rates of illiteracy and ignorance;

8. Low level of awareness of the benefits of modern communications, resulting in reduced demand in some regions; 
9. Lack of funding from both the public and private sectors.

However, in Sri Lanka, the most important challenges in ICT/ICT development in rural and remote areas, according to [23], were summarized as follows:

- High operating costs construction: Because far and remote areas lack the proper infrastructure to support the communication process, they need high financial support to overcome this problem. This is because of the related issues of transportation and investment related to energy and construction required to run this advanced equipment In addition, there might be a need to use electric generators which further raises the cost.

- Low population and low average revenue potential: Low population density and consequently lower average revenue are associated with most remote areas. This is because of social and economic reasons in those regions.

- Lack of energy supply: Lack of energy supply for battery and generator stores is critical; emphasis should be placed on alternative investments in areas such as solar power, wind farms, and others, which further increase the financial impact.

- Lack of technical personnel: Telecommunicationsrelated investments require highly competent technicians to operate skillful tasks, which is something that is not always available in rural areas. This will add further burden on the budget of investment in these areas.

\section{Recommendations:}

This study recommends the following:

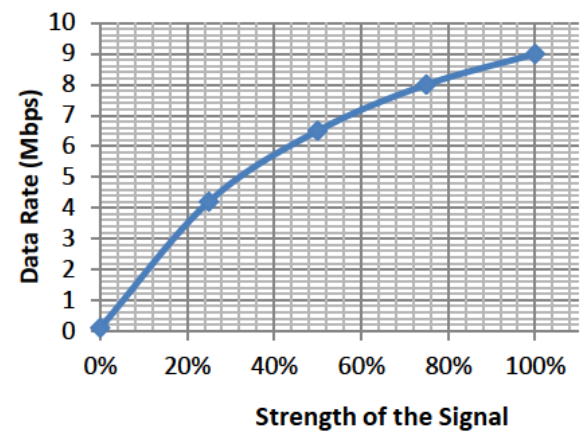

Figure 6: Relation between data rate and different signal strengths.
- Considering efficient ways of building up wireless networks by using a sensor to detect the need for a high-strength signal (as shown in Figure 6).

- Investigating other causes that affect the signal strength of the wireless networks that provide internet access in remote areas, and any other factors that help to extend networks with weak signals.

\section{Conclusion}

There is an over-reliance on smart phones and devices because this is the most convenient form of communication nowadays. Smart phones can access internet services via wireless connectivity. As a result, people are highly reliant on them when sending and receiving various types of information.

This study focused on how to increase the signal strength of wireless networks and how to overcome the most prominent challenges in communication in remote areas. The results show that many steps and observations help people increase the signal strength of the network, and that there are many challenges and difficulties in developing communication in remote areas, the most prominent of which relates to the geographical location.

Acknowledgement: The author is grateful to the University of Mosul, Faculty of Engineering, Department of Computer Engineering for providing the facilities in carrying out this work.

\section{References}

[1] Xu G., Shi Y., Sun X., Shen W., Internet of Things in Marine Environment Monitoring: A Review. Sensors, 2019, 19(7), 1711.

[2] Kamal Z. E. H., Salahuddin M.A., Introduction to Wireless Sensor Networks. In: Benhaddou D., Al-Fuqaha A. (eds) Wireless Sensor and Mobile Ad-Hoc Networks, Springer, New York, NY, 2015

[3] Oteyo I. N., Toili M. E. M., Improving Specimen Labelling and Data Collection in Bio-science Research using Mobile and Web Applications, Open Computer Science, 2020, 10.1, 1-16.

[4] Gölpek, F., Service Sector and Technological Developments, Procedia - Social and Behavioral Sciences, 2015, 181. 10.1016/j.sbspro.2015.04.873.

[5] Hamad M. A., Eltahir M. E. S., Ali A. E. M., \& Hamdan A. M., Efficiency of using smart-mobile phones in accessing agricultural information by smallholder farmers in North Kordofan - Sudan, 2018, Available at SSRN 3240758.

[6] Khanna A., Kaur S., Evolution of Internet of Things (IoT) and its significant impact in the field of Precision Agriculture, Computers and electronics in agriculture, 2019, 157, 218-231. 
[7] Mutahar B., Al-Zzalab A., Mobile phone as media intermediary, Academic edu, 2015

[8] Shoukat S., Cell phone addiction and psychological and physiological health in adolescents, EXCLI journal, 2019, 18, 47-50.

[9] Mudumbai R., Hespanha J., Madhow U., \& Barriac G., Scalable feedback control for distributed beamforming in sensor networks, Proceedings of International Symposium on Information Theory, (2005), ISIT 2005, 137-141. IEEE.

[10] Voigt T., Bor M., Roedig U., \& Alonso J., Mitigating internetwork interference in LoRa networks, 2016, arXiv preprint arXiv:1611.00688.

[11] Pardo-Castellote G., \& Schneider S., The network data delivery service: Real-time data connectivity for distributed control applications, Proceedings of the 1994 IEEE International Conference on Robotics and Automation, 1994, 2870-2876. IEEE.

[12] Almadani B., RTPS middleware for real-time distributed industrial vision systems, Proceedings of the 11th IEEE International Conference on Embedded and Real-Time Computing Systems and Applications (RTCSA'05), 2005, 361-364. IEEE.

[13] Adediran Y., Faruk N., Opadiji J.F., Bello O., On Issues and Challenges of Rural Telecommunications Access in Nigeria, African Journal of Research in Mathematics, Science and Technology Education, 2016, 3(2), 16-26.

[14] Dubale T. E., Telecommunication in Ethiopia, Proceedings of Multi-year expert meeting on services, development, and trade: The regulatory and institutional dimension, 2010, 17-19

[15] Garcia E., Elbeltagi I., \& Bugliolo M., Introducing 4G mobile networks: implications for UK higher education, The International Journal of Information and Learning Technology, 2015, 32 (2), 124 -135 .
[16] Dahiya M., Evolution of Wireless LAN in Wireless Networks, International Journal on Computer Science and Engineering, 2017, 9, 109-113.

[17] Didenko N., McNay K., Method and Apparatus For Monitoring Mobile Communication Networks, U.S. Patent Application, 2015, $14 / 139,404$

[18] Chou C. M., Hsu C. C., Johansson P. J. M., U.S. Patent No. 10,104,612. Washington, DC: U.S. Patent and Trademark Office, 2018

[19] Freeman J., Park S., Middleton C., Technological literacy and interrupted internet access, Information, Communication \& Society, 2019, 0 (0), 1-18.

[20] Manshaei M. H., Hubaux J. P., Hands-On Exercises: IEEE 802.11 Standard. Tech. rep., École Polytechnique Fédérale de Lausanne, Lausanne, Switzerland, 2010

[21] Rajab S. A., Balid W., \& Refai H. H., Comprehensive study of spectrum occupancy for $802.11 \mathrm{~b} / \mathrm{g} / \mathrm{n}$ homogeneous networks, Proceedings of 2015 IEEE International Instrumentation and Measurement Technology Conference (I2MTC), 2015, 1741-1746, IEEE.

[22] Samuel,K. J., \& Ayeni B., A GIS-based analysis of geographical accessibility to shared information and communications technology (ICT) infrastructure in a remote region of Nigeria, African Journal of Science, Technology, Innovation and Development, 2019, 11(1), 121-129.

[23] ITU. Democratic Socialist Republic of Sri Lanka. International Telecommunication Union ITU, 2016 